\title{
Improved 18S and 28S rDNA primer sets for NGS-based parasite detection
}

\begin{abstract}
Asuka Kounosu, Kazunori Murase, Akemi Yoshida, Haruhiko Maruyama \& Taisei Kikuchi $\mathbb{C}^{*}$
The development and application of next-generation sequencing (NGS) have enabled comprehensive analyses of the microbial community through extensive parallel sequencing. Current analyses of the eukaryotic microbial community are primarily based on polymerase chain reaction amplification of $18 \mathrm{~S}$ rRNA gene ( $r D N A$ ) fragments. We found that widely-used $18 \mathrm{~S}$ rDNA primers can amplify numerous stretches of the bacterial 16S rRNA gene, preventing the high-throughput detection of rare eukaryotic species, particularly in bacteria-rich samples such as faecal material. In this study, we employed in silico and NGS-based analyses of faecal samples to evaluated the existing primers targeting eukaryotic $18 \mathrm{~S}$ and $28 \mathrm{~S}$ rDNA in terms of avoiding bacterial read contamination and improving taxonomic coverage for eukaryotes, with a particular emphasis on parasite taxa. Our findings revealed that newly selected primer sets could achieve these objectives, representing an alternative strategy for NGS.
\end{abstract}

Next-generation sequencing (NGS) using the 16S rRNA gene (16S rDNA) has been widely used to examine bacterial diversity ${ }^{1,2}$. In our previous study, we applied NGS using the $18 \mathrm{~S}$ rRNA gene (18S rDNA) to analyse eukaryotic parasite diversity ${ }^{3,4}$. Compared with conventional methods which rely on host dissections and/or microscopic observations, NGS-based methods are easy and sufficiently sensitive for high-throughput analyses ${ }^{3,4}$.

$18 \mathrm{~S}$ rDNA has been widely used for the identification and diversity analyses of eukaryotes because it is well conserved among species and it contains variable regions ${ }^{5,6}$. Within $18 \mathrm{~S}$ rDNA, hypervariable regions 4 (V4) and 9 (V9) are currently the popular options for NGS-based analyses ${ }^{5-8}$. The Earth Microbiome Project (EMP), which aims to construct a global catalogue of the uncultured microbial diversity on the Earth ${ }^{9}$, recommends the use of primers that amplify a short fragment (approximately $150 \mathrm{bp}$ ) containing the V9 region of $18 \mathrm{~S}$ rDNA for eukaryote analyses. As with those studies using PCR, primer selection is a critical factor for successful NGS-based analyses because non-universal primers are subject to taxonomic biases. Along with the elongation of the read length by the Illumina sequencer, some recent studies seeking to develop improved primer sets which amplify longer fragments have compared $18 \mathrm{~S}$ rDNA among all eukaryotes ${ }^{5,6}$ or specific taxa ${ }^{8}$ via in silico sequence analysis and identified conserved regions best suited for amplifying the hypervariable regions. However, although those primer sets were designed to amplify eukaryotic $18 \mathrm{~S}$ rDNA fragments, several bacterial 16S rDNA fragments were also amplified ${ }^{10-12}$, indicating their poor specificity. Specifically, for bacteria-rich samples, such as faecal material, bacterial read contamination represents a critical drawback, preventing the detection of rare eukaryotic species. In addition, refined classification of the detected reads to the genus or species level is often difficult using $18 \mathrm{~S}$ rDNA primers because the amplicon sequence does not represent sufficient sequence diversity to distinguish closely related genera or species. Other genomic regions, such as the large subunit (LSU) of rDNA, which varies from $25 \mathrm{~S}$ to $28 \mathrm{~S}$ in size depending on the species (in this article we use " $28 \mathrm{~S}$ rDNA" to refer eukaryotic LSU), or the ITS regions of rDNA, which show higher diversity than $18 \mathrm{~S}$ rDNA, represent alternative targets for PCR amplification $^{13,14}$.

In this study, we sought to identity primer sets that provide high taxonomic resolution and less bacterial read contamination to investigate eukaryotic microbial diversity with a particular emphasis on parasitic taxa. Primer screening was performed using $18 \mathrm{~S}$ and $28 \mathrm{~S}$ rDNA via in silico sequence analyses, and selected primers were further evaluated for sensitivity, specificity, taxonomic discrimination capacity, amplification efficiency and reproducibility via quantitative PCR (qPCR) and NGS analyses of faecal samples obtained from parasite-infected animals. 


\begin{tabular}{|c|c|c|c|c|c|c|c|c|c|c|c|c|c|}
\hline \multirow{4}{*}{$\begin{array}{l}\text { Taxonomy }^{\mathrm{a}} \\
\begin{array}{l}\text { Nematoda } \\
(2169)\end{array}\end{array}$} & \multirow{4}{*}{\begin{tabular}{|l|}
$\begin{array}{l}\text { Representative } \\
\text { species }\end{array}$ \\
$\begin{array}{l}\text { Roundworm, } \\
\text { Filaria }\end{array}$ \\
\end{tabular}} & \multicolumn{2}{|c|}{$\operatorname{EMP}(1391 \mathrm{~F} / \mathrm{EukBr})^{\mathrm{b}, \mathrm{c}}$} & \multicolumn{2}{|c|}{$563 \mathrm{~F} / 1132 \mathrm{R}^{\mathrm{c}}$} & \multicolumn{2}{|c|}{$574 \mathrm{~F} / 952 \mathrm{R}^{\mathrm{c}}$} & \multicolumn{2}{|c|}{$574 * \mathrm{~F} / 952 \mathrm{R}^{\mathrm{c}}$} & \multicolumn{2}{|c|}{$616 * F / 1132 R^{c}$} & \multicolumn{2}{|c|}{$1183 F / 1631 R^{c}$} \\
\hline & & \multicolumn{2}{|l|}{ V9 } & \multicolumn{2}{|l|}{ V4-V5 } & \multicolumn{2}{|l|}{ V4-V5 } & \multicolumn{2}{|l|}{ V4-V5 } & \multicolumn{2}{|l|}{ V4-V5 } & \multicolumn{2}{|l|}{ V7-V8 } \\
\hline & & \multicolumn{2}{|l|}{145 bp } & \multicolumn{2}{|l|}{$569 \mathrm{bp}$} & \multicolumn{2}{|l|}{$378 \mathrm{bp}$} & \multicolumn{2}{|l|}{378 bp } & \multicolumn{2}{|l|}{ 516bp } & \multicolumn{2}{|l|}{449 bp } \\
\hline & & 88.2 & $(551 / 625)$ & 95.9 & $(2072 / 2160)$ & 42.0 & $(909 / 2163)$ & 93.2 & $(2015 / 2163)$ & 96.0 & $(2080 / 2166)$ & 88.0 & $(1824 / 2072)$ \\
\hline $\begin{array}{l}\text { Platyhelminthes } \\
\text { (1963) }\end{array}$ & Tapeworm, Fluke & 81.8 & $(306 / 374)$ & 88.4 & $(1713 / 1937)$ & 89.2 & $(1734 / 1945)$ & 88.9 & $(1729 / 1945)$ & 88.7 & $(1727 / 1947)$ & 90.1 & $(1713 / 1901)$ \\
\hline $\begin{array}{l}\text { Acanthocephala } \\
\text { (63) }\end{array}$ & $\begin{array}{l}\text { Spiny-headed } \\
\text { worm }\end{array}$ & 100.0 & $(26 / 26)$ & 95.2 & $(60 / 63)$ & 0.0 & $(0 / 63)$ & 0.0 & $(0 / 63)$ & 93.7 & $(59 / 63)$ & 0.0 & $(0 / 62)$ \\
\hline Coccidia (671) & Coccidium & 96.0 & $(168 / 175)$ & 97.9 & $(656 / 670)$ & 96.7 & $(649 / 671)$ & 96.7 & $(649 / 671)$ & 97.2 & $(652 / 671)$ & 90.8 & $(444 / 489)$ \\
\hline $\begin{array}{l}\text { Cryptosporida } \\
\text { (55) }\end{array}$ & Cryptosporidium & 97.3 & $(36 / 37)$ & 94.5 & $(52 / 55)$ & 96.4 & $(53 / 55)$ & 96.4 & $(53 / 55)$ & 92.7 & $(51 / 55)$ & 100.0 & $(53 / 53)$ \\
\hline $\begin{array}{l}\text { Haemosporidia } \\
\text { (149) }\end{array}$ & Plasmodium & 87.5 & $(63 / 72)$ & 0.0 & $(0 / 148)$ & 98.0 & $(145 / 148)$ & 98.0 & $(145 / 148)$ & 0.0 & $(0 / 149)$ & 0.0 & $(0 / 95)$ \\
\hline Fornicata (21) & Giardia & 100.0 & $(7 / 7)$ & 100.0 & $(21 / 21)$ & 23.8 & $(5 / 21)$ & 33.3 & $(7 / 21)$ & 71.4 & $(15 / 21)$ & 30.0 & $(6 / 20)$ \\
\hline $\begin{array}{l}\text { Discicristata } \\
(909)\end{array}$ & $\begin{array}{l}\text { Trypanosoma, } \\
\text { Leishmania }\end{array}$ & 92.2 & $(306 / 332)$ & 93.0 & $(806 / 867)$ & 68.0 & $(608 / 894)$ & 69.9 & (663/949) & 71.7 & $(640 / 893)$ & 86.2 & $(698 / 810)$ \\
\hline Parabasalia (303) & Trichomonas & 77.0 & $(47 / 61)$ & 98.0 & $(297 / 303)$ & 0.0 & $(0 / 303)$ & 44.2 & $(134 / 303)$ & 19.8 & $(60 / 303)$ & 0.0 & $(0 / 299)$ \\
\hline Entamoebida (4) & Entamoeba & 100.0 & $(3 / 3)$ & 100.0 & $(4 / 4)$ & 100.0 & $(4 / 4)$ & 100.0 & $(4 / 4)$ & 100.0 & $(4 / 4)$ & 0.0 & $(0 / 3)$ \\
\hline $\begin{array}{l}\text { Longamoebia } \\
\text { (158) }\end{array}$ & Acanthamoeba & 73.6 & $(39 / 53)$ & 87.4 & $(125 / 143)$ & 89.0 & $(130 / 146)$ & 90.4 & $(132 / 146)$ & 88.0 & $(132 / 150)$ & 89.5 & $(119 / 133)$ \\
\hline Fungi (15101) & & 56.4 & (3076/5458) & 96.6 & $(14501 / 15006)$ & 45.3 & $(1434 / 3164)$ & 95.6 & $(14412 / 15071)$ & 93.7 & $(14156 / 15100)$ & 91.9 & $(13132 / 14290)$ \\
\hline $\begin{array}{l}\text { Bacteria } \\
(575268)\end{array}$ & & 37.6 & $(34016 / 90407)$ & 89.9 & $(516999 / 58262)$ & $<0.1$ & $(12 / 575261)$ & $<0.1$ & $(14 / 575247)$ & $<0.1$ & $(252 / 575265)$ & $<0.1$ & $(1 / 558088)$ \\
\hline $\begin{array}{l}\text { Euteleostomi } \\
(1091)\end{array}$ & Mammal $^{\mathrm{d}}$ & $55.0^{\mathrm{h}, \mathrm{m}}$ & $(382 / 694)$ & $75.5^{\mathrm{h}, \mathrm{m}}$ & $(816 / 1081)$ & $72.5^{\mathrm{h}, \mathrm{m}}$ & $(784 / 1081)$ & $61.6^{\mathrm{h}, \mathrm{m}}$ & $(788 / 1081)$ & $75.0^{\mathrm{h}, \mathrm{m}}$ & $(815 / 1087)$ & $62.5^{\mathrm{h}, \mathrm{m}}$ & $(599 / 958)$ \\
\hline
\end{tabular}

Table 1. List of primer sets targeting the $18 \mathrm{~S}$ rRNA gene and their coverage in 14 taxonomic groups. Primer sets were tested for matches to sequences in the SILVA database (v.132) using TestPrime under the following parameters: maximum number of mismatches of four bases and length of 0 -mismatch at the $3^{\prime}$ end of three bases). TestPrime computes coverages for each taxonomic group by running in silico PCR on the SILVA database via sorting database sequences into "match", "mismatch" and "nodata (sequences not covering the primer match position)". The frequencies of "match" sequences among "match" and "mismatch" sequences are

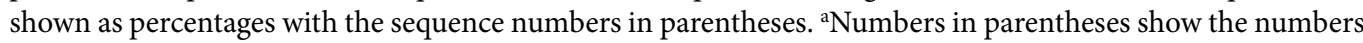
of sequences available in the SILVA database. Please note these numbers are not always the denominators

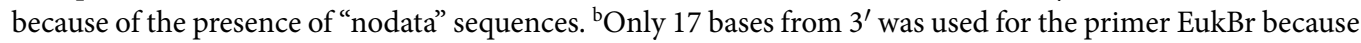
many sequences in the SILVA database lacks the corresponding $5^{\prime}$ region. ${ }^{c}$ Target variable regions and amplicon sizes based on the S. cerevisiae rRNA gene (NC_001144) are shown below the primer names. ${ }^{\mathrm{d}}$ Primer match to humans and mice is indicated by superscripts $h$ and $m$ on the values, respectively.

\section{Results}

In silico screening. For NGS-based analyses of eukaryote diversity, previous studies have mostly used 1391F/ EukBr, as recommended by EMP ${ }^{10,15-17}$, or $563 \mathrm{~F} / 1132 \mathrm{R}^{18-22}$, which targets the V9 or V4-5 regions of $18 \mathrm{~S}$ rDNA, respectively. In this article, these two primer sets are referred to as 'conventional primer sets' and used as comparators. To identify primer pairs that can efficiently detect a wide variety of parasites while avoiding bacterial DNA amplification for use in NGS-based parasite detection, we screened all possible $18 \mathrm{~S}$ and $28 \mathrm{~S}$ rDNA primers. Some previous studies have extensively tested $18 \mathrm{~S}$ rDNA primers in silico to design universal eukaryotic primers to be used as standards for NGS-based analyses of eukaryote diversity ${ }^{5,6,13}$. Therefore, we used these recommended $18 \mathrm{~S}$ rDNA primer sets to re-evaluate for detection of parasitic taxa groups (Table 1; Supplementary Table S1). For 28S rDNA, we retrieved the possible universal primers $(\mathrm{n}=52)$ (Supplementary Table S1) from previous reports ${ }^{13,23,24}$ and screened them based on their melting temperatures (Tm) and amplicon sizes (Materials and Methods), yielding 13 primer pairs (Table 2).

We then evaluated those primer pairs based on their sequence identity with eukaryotic parasites, fungi and bacteria using the SILVA non-redundant sequence dataset (Tables 1 and 2). The EMP primer set (1391F/EukBr) can be used to detect a wide variety of parasitic taxa, exhibiting 43.4-66.7\% coverage in majority of the tested taxa, excluding Nematoda, Platyhelminthes, Longamoebia and Fungi. The other conventional primer set 563F/1132R exhibited higher coverage than the EMP primer set ( $\geq 87.4 \%$ excluding Haemosporodia). However, these two primer sets showed similarity with bacterial $16 \mathrm{~S}$ rDNA sequences ( $13.0 \%$ and $89.9 \%$, respectively). Although the other $18 \mathrm{~S}$ primer sets demonstrated lower taxonomic coverage for eukaryotes than $563 \mathrm{~F} / 1132 \mathrm{R}$, they appeared to amplify less bacterial rDNA. For instance, 574F/952R showed low coverage for Nematoda, Fornicata and Parabasalia. Moreover, 574F/952R, 574*F/952R and 1183F/1631R showed low coverage for Acanthocephala, while $1183 \mathrm{~F} / 1631 \mathrm{R}$ showed low coverage for Parabasalia, Haemosporidia and Entamoebida, despite their low similarities to bacterial sequences (coverage $<0.1 \%$ ). These tendencies were also observed when we used strict or mild parameters for taxonomic coverage evaluations (Tables S2 and S3). Based on these results, $616 * \mathrm{~F} / 1132 \mathrm{R}$ and $1183 \mathrm{~F} / 1631 \mathrm{R}$ were selected for further evaluation as the best primer sets for the $18 \mathrm{~S} \mathrm{~V} 4-5$ and V7-8 regions, respectively. 


\begin{tabular}{|c|c|c|c|c|c|c|c|c|c|c|c|c|c|}
\hline \multirow{4}{*}{$\begin{array}{l}\text { Taxonomy } \\
\text { Nematoda (659) }\end{array}$} & \multirow{4}{*}{\begin{tabular}{|l} 
Representative \\
species
\end{tabular}} & \multicolumn{2}{|c|}{ DM568F/RM2R $^{\mathrm{b}}$} & \multicolumn{2}{|c|}{ DM568F/GA13R } & \multicolumn{2}{|c|}{ DM568F/RM3R $^{\mathrm{b}}$} & \multicolumn{2}{|c|}{ RM2F/RM3R ${ }^{b}$} & \multicolumn{2}{|c|}{ RM2F/GA15R ${ }^{b}$} & \multicolumn{2}{|c|}{ GA12F/RM4R $^{\mathrm{b}}$} \\
\hline & & \multicolumn{2}{|c|}{ D3-D4 } & \multicolumn{2}{|c|}{ D3-D4 } & \multicolumn{2}{|c|}{ D3-D5 } & \multicolumn{2}{|c|}{ D4-D5 } & \multicolumn{2}{|c|}{ D4-D5 } & \multicolumn{2}{|c|}{ D4-D6 } \\
\hline & & \multicolumn{2}{|l|}{284 bp } & \multicolumn{2}{|c|}{$327 \mathrm{bp}$} & \multicolumn{2}{|l|}{500 bp } & \multicolumn{2}{|l|}{$236 \mathrm{bp}$} & \multicolumn{2}{|l|}{$369 \mathrm{bp}$} & \multicolumn{2}{|l|}{$507 \mathrm{bp}$} \\
\hline & & 62.1 & $(339 / 546)$ & 42.5 & $(232 / 546)$ & 62.1 & $(339 / 546)$ & 94.7 & $(551 / 582)$ & 54.8 & $(319 / 582)$ & 92.9 & $(546 / 588)$ \\
\hline $\begin{array}{l}\text { Platyhelminthes } \\
\text { (571) }\end{array}$ & Tapeworm, Fluke & 88.2 & $(439 / 498)$ & 84.9 & $(423 / 498)$ & 91.4 & $(455 / 498)$ & 95.2 & $(496 / 521)$ & 23.2 & $(121 / 521)$ & 97.3 & $(510 / 524)$ \\
\hline $\begin{array}{l}\text { Acanthocephala } \\
\text { (56) }\end{array}$ & $\begin{array}{l}\text { Spiny-headed } \\
\text { worm }\end{array}$ & 94.6 & $(53 / 56)$ & 69.6 & $(39 / 56)$ & 94.6 & $(53 / 56)$ & 94.6 & $(53 / 56)$ & 28.6 & $(16 / 56)$ & 100.0 & $(56 / 56)$ \\
\hline Coccidia (76) & Coccidium & 90.4 & $(66 / 73)$ & 91.8 & $(67 / 73)$ & 90.4 & $(66 / 73)$ & 91.9 & $(68 / 74)$ & 83.8 & $(62 / 74)$ & 93.2 & $(69 / 74)$ \\
\hline Cryptosporida (1) & Cryptosporidium & 100.0 & $(1 / 1)$ & 100.0 & $(1 / 1)$ & 100.0 & $(1 / 1)$ & 100.0 & $(1 / 1)$ & 100.0 & $(1 / 1)$ & 100.0 & $(1 / 1)$ \\
\hline $\begin{array}{l}\text { Haemosporidia } \\
\text { (115) }\end{array}$ & Plasmodium & 100.0 & $(89 / 89)$ & 0.0 & $(0 / 89)$ & 95.5 & $(85 / 89)$ & 94.9 & $(93 / 98)$ & 87.8 & $(86 / 98)$ & 0.0 & $(0 / 101)$ \\
\hline Fornicata (5) & Giardia & 20.0 & $(1 / 4)$ & 0.0 & $(0 / 5)$ & 0.0 & $(0 / 5)$ & 0.0 & $(0 / 5)$ & 20.0 & $(1 / 4)$ & 40.0 & $(2 / 5)$ \\
\hline Discicristata (35) & $\begin{array}{l}\text { Trypanosoma, } \\
\text { Leishmania }\end{array}$ & 67.7 & $(21 / 31)$ & 61.3 & $(19 / 31)$ & 41.9 & $(13 / 31)$ & 40.4 & $(13 / 32)$ & 75.0 & $(24 / 32)$ & 100.0 & $(33 / 33)$ \\
\hline Parabasalia (20) & Trichomonas & 100.0 & $(17 / 17)$ & 94.1 & $(16 / 17)$ & 0.0 & $(0 / 17)$ & 0.0 & $(0 / 18)$ & 61.1 & $(11 / 18)$ & 5.6 & $(1 / 18)$ \\
\hline Entamoebida (9) & Entamoeba & 100.0 & $(5 / 5)$ & 0.0 & $(0 / 5)$ & 0.0 & $(0 / 5)$ & 0.0 & $(0 / 8)$ & 0.0 & $(0 / 8)$ & 0.0 & $(0 / 8)$ \\
\hline Longamoebia (1) & Acanthamoeba & 100.0 & $(1 / 1)$ & 100.0 & $(2 / 2)$ & 100.0 & $(1 / 1)$ & 100.0 & $(2 / 2)$ & 100.0 & $(1 / 1)$ & 100.0 & $(1 / 1)$ \\
\hline Fungi (3671) & & 81.8 & $(2876 / 3515)$ & 93.2 & $(3277 / 3515)$ & 94.1 & $(3308 / 3515)$ & 93.6 & $(3347 / 3576)$ & 85.1 & $(3044 / 3576)$ & 95.5 & $(3416 / 3578)$ \\
\hline Bacteria (124805) & & $<0.1$ & $(12 / 122765)$ & $<0.1$ & $(5 / 122772)$ & $<0.1$ & $(9 / 122777)$ & $<0.1$ & $(9 / 123872)$ & $<0.1$ & $(22 / 123872)$ & $<0.1$ & $(5 / 124043)$ \\
\hline Euteleostomi (445) & $\mathrm{Mammal}^{\mathrm{c}}$ & $74.7^{\mathrm{h}, \mathrm{m}}$ & $(236 / 316)$ & $75.3^{\mathrm{h}, \mathrm{m}}$ & $(238 / 316)$ & $77.2^{\mathrm{h}, \mathrm{m}}$ & $(244 / 316)$ & $79.5^{\mathrm{h}, \mathrm{m}}$ & $(303 / 381)$ & $75.3^{\mathrm{h}, \mathrm{m}}$ & $(287 / 381)$ & $82.7^{\mathrm{h}, \mathrm{m}}$ & $(324 / 392)$ \\
\hline \multicolumn{2}{|l|}{ RM3F/RM4R ${ }^{b}$} & \multicolumn{2}{|c|}{ GA14F/RM4R } & \multicolumn{2}{|c|}{ GA18F/RM7R $^{\text {b }}$} & \multicolumn{2}{|c|}{ GA18F/RM8R ${ }^{\mathbf{b}}$} & \multicolumn{2}{|c|}{ GA20F/RM7R $^{\mathbf{b}}$} & GA20F/ & RM8R $^{\text {b }}$ & GA20F/ & RM9R $^{\text {b }}$ \\
\hline D6 & & D6 & & D8 & & D8 & & D8 & & D8-D9 & & D8-D9 & \\
\hline 333 bp & & $202 \mathrm{bp}$ & & $424 \mathrm{bp}$ & & $572 \mathrm{bp}$ & & $348 \mathrm{bp}$ & & $406 \mathrm{bp}$ & & $505 \mathrm{bp}$ & \\
\hline 92.5 & $(564 / 610)$ & 95.5 & $(592 / 620)$ & 95.9 & $(626 / 653)$ & 95.8 & $(619 / 646)$ & 97.7 & $(638 / 653)$ & 97.8 & $(632 / 646)$ & \begin{tabular}{|l|l|}
98.0 \\
\end{tabular} & $(632 / 645)$ \\
\hline 97.8 & $(523 / 535)$ & 96.7 & $(526 / 544)$ & 86.3 & $(465 / 539)$ & 89.0 & $(471 / 529)$ & 91.7 & $(493 / 539)$ & 94.5 & $(500 / 529)$ & 96.6 & $(510 / 528)$ \\
\hline 100.0 & $(56 / 56)$ & 35.7 & $\begin{array}{l}(20 / 56) \\
\end{array}$ & 96.4 & $(54 / 56)$ & 100.0 & $(56 / 56)$ & 96.4 & $(54 / 56)$ & 100.0 & $(56 / 56)$ & 100.0 & $(56 / 56)$ \\
\hline 91.9 & $(68 / 74)$ & 91.9 & $(68 / 74)$ & 97.1 & $(68 / 70)$ & 95.5 & $(64 / 67)$ & 98.6 & $(69 / 70)$ & 97.0 & $(65 / 67)$ & 98.5 & $(65 / 66)$ \\
\hline 100.0 & $(1 / 1)$ & 100.0 & $(1 / 1)$ & 100.0 & $(1 / 1)$ & 100.0 & $(1 / 1)$ & 100.0 & $(1 / 1)$ & 100.0 & $(1 / 1)$ & 100.0 & $(1 / 1)$ \\
\hline 95.2 & $(99 / 104)$ & 98.1 & $(104 / 106)$ & 61.1 & $(69 / 113)$ & 61.6 & $(69 / 112)$ & 92.0 & $(104 / 113)$ & 97.3 & $(109 / 112)$ & 97.3 & $(109 / 112)$ \\
\hline 20.0 & $(1 / 5)$ & 60.0 & $(3 / 5)$ & 0.0 & $(0 / 5)$ & 0.0 & $(0 / 5)$ & 100.0 & $(5 / 5)$ & 100.0 & $(5 / 5)$ & 100.0 & $(5 / 5)$ \\
\hline 57.1 & $(20 / 35)$ & 100.0 & $(35 / 35)$ & 48.6 & $(17 / 35)$ & 45.7 & $(16 / 35)$ & 62.9 & $(22 / 35)$ & 60.0 & $(21 / 35)$ & 62.9 & $(22 / 35)$ \\
\hline 0.0 & $(0 / 20)$ & 0.0 & $(0 / 20)$ & 0.0 & $(0 / 20)$ & 36.8 & $(7 / 19)$ & 0.0 & $(0 / 20)$ & 94.7 & $(18 / 19)$ & 94.7 & $(18 / 19)$ \\
\hline 100.0 & $(9 / 9)$ & 100.0 & $(9 / 9)$ & 0.0 & $(0 / 9)$ & 0.0 & $(0 / 9)$ & 0.0 & $(0 / 9)$ & 0.0 & $(0 / 9)$ & 0.0 & $(0 / 9)$ \\
\hline 100.0 & $(1 / 1)$ & 100.0 & $(1 / 1)$ & 100.0 & $(2 / 2)$ & 100.0 & $(1 / 1)$ & 100.0 & $(1 / 1)$ & 100.0 & $(1 / 1)$ & 100.0 & $(1 / 1)$ \\
\hline 94.7 & $(3425 / 3617)$ & 95.5 & $(3469 / 3631)$ & 90.7 & $(2943 / 3244)$ & 88.6 & $(2839 / 3205)$ & 95.2 & $(3087 / 3244)$ & 92.5 & $(2966 / 3205)$ & 93.8 & $(3006 / 3205)$ \\
\hline$<0.1$ & $(5 / 124626)$ & $<0.1$ & $(6 / 124788)$ & $<0.1$ & $(13 / 124775)$ & $<0.1$ & $(10 / 124065)$ & $<0.1$ & $(13 / 124775)$ & $<0.1$ & $(10 / 124065)$ & $<0.1$ & $(23 / 124041)$ \\
\hline $83.3^{\mathrm{h}, \mathrm{m}}$ & $(340 / 408)$ & $82.3^{\mathrm{h}, \mathrm{m}}$ & $(340 / 413)$ & $74.2^{\mathrm{h}, \mathrm{m}}$ & $(316 / 426)$ & $75.6^{\mathrm{h}, \mathrm{m}}$ & $(304 / 402)$ & $72.3^{\mathrm{h}, \mathrm{m}}$ & $(308 / 426)$ & $73.6^{\mathrm{h}, \mathrm{m}}$ & $(296 / 402)$ & $75.3^{\mathrm{h}, \mathrm{m}}$ & $(302 / 401)$ \\
\hline
\end{tabular}

Table 2. List of primer sets targeting the $28 \mathrm{~S}$ rRNA gene and their coverages in 14 taxonomic groups. Primer sets were tested for matches to sequences in the SILVA database (v.132) using TestPrime under the following parameters: maximum number of mismatches of four bases and length of 0 -mismatch at the $3^{\prime}$ end of three bases). TestPrime computes coverages for each taxonomic group by running in silico PCR on the SILVA database via sorting database sequences into "match", "mismatch" and "nodata (sequences not covering the primer match position)". The frequencies of "match" sequences among "match" and "mismatch" sequences are

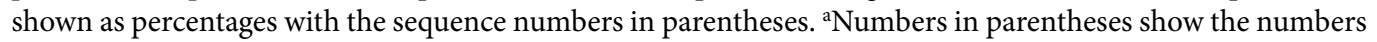
of sequences available in the SILVA database. Please note these numbers are not always the denominators because of the presence of "nodata" sequences. 'Target variable regions and amplicon sizes based on the $S$. cerevisiae rRNA gene (NC_001144) are shown below the primer names. ${ }^{c}$ Primer match to humans and mice is indicated by superscripts $h$ and $m$ on the values, respectively.

Data for the 28S rDNA primers are summarised in Tables 2, S2 and S3. All 28S primer sets showed low similarity with bacterial sequences. The taxonomic coverage for some eukaryotic parasites was variable, especially for some protozoan groups including Haemosporidia, Fornicata, Parabasalia and Entamoebida. On the other hand, the coverage for the other parasitic taxa were not very different, although the primer sets designed for the D3-D5 regions showed low coverage for Nematoda, Platyhelminthes and Discicristata. Among the five primer sets designed based on the D8-D9 regions, GA20F/RM8R, RM7F/RM9R and GA20F/RM9R exhibited wide taxonomic coverage except for Entamoebida. Based on these results, we selected seven 28S primer sets, namely DM568F/RM2R, RM2F/RM3R, RM3F/RM4R, GA12F/RM4R, GA20F/RM7R, GA20F/RM8R and GA20F/ RM9R, for further evaluation. 


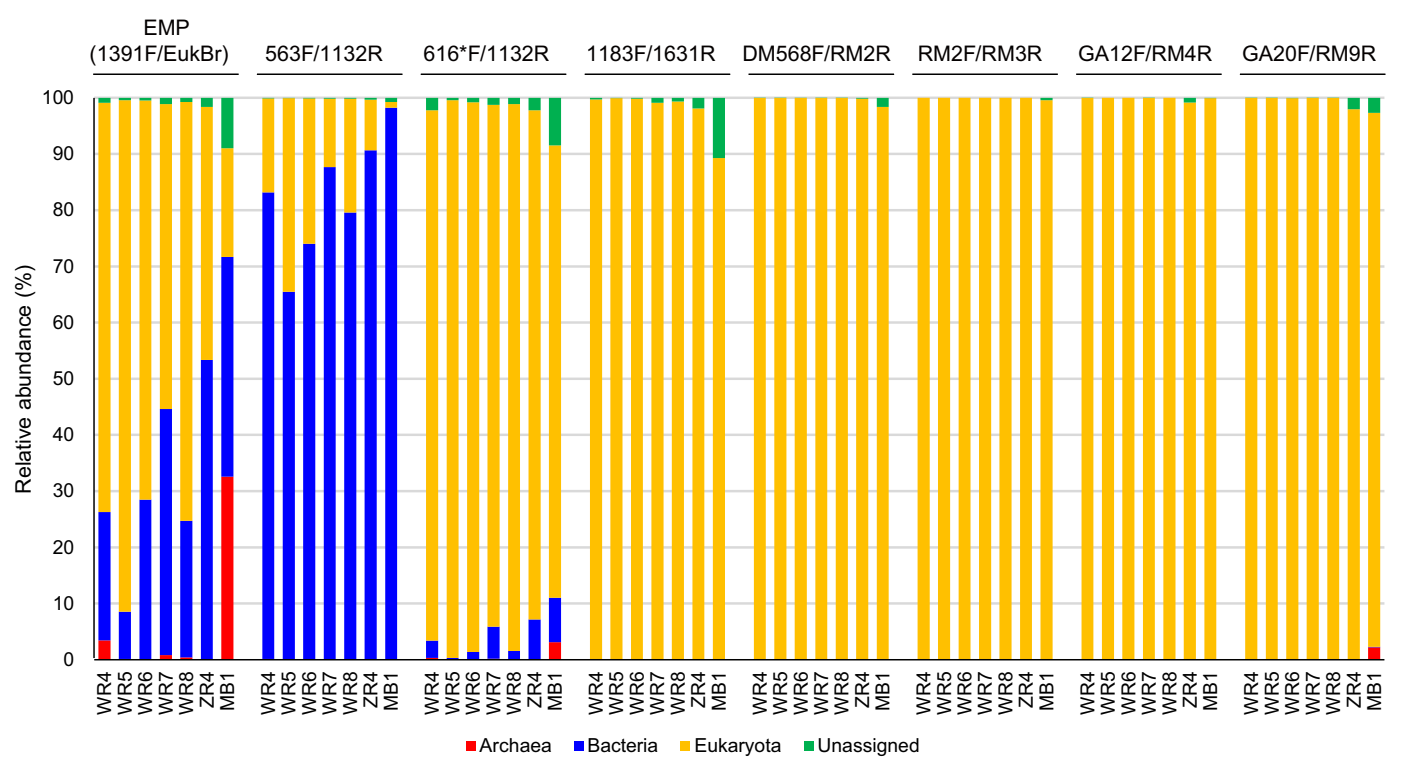

Figure 1. Domain-level classification of total Illumina reads retrieved from PCR amplicons using eight primer sets and seven faecal samples.

All the $18 \mathrm{~S}$ and $28 \mathrm{~S}$ primers tested showed high coverages $(>60 \%)$ for Euteleostomi which includes their possible hosts. In particular, human and mouse DNA are likely to be amplified by all primers tested (Tables 1 and 2).

qPCR. To confirm whether the selected primer sets could efficiently amplify eukaryotic rDNA without dimer or hairpin structure generation, we performed qPCR using C. elegans DNA as representative eukaryote DNA because all selected primers displayed $100 \%$ sequence similarity with C. elegans rRNA. 18S rDNA from $0.1 \mathrm{ng}$ of C. elegans genomic DNA (final concentration, $0.01 \mathrm{ng} / \mu \mathrm{l}$ ), which corresponds to $\sim 200,000$ copies of rRNA, was amplified at $\sim 21$ cycles (mean $\mathrm{Ct} \pm \mathrm{SD}=21.28 \pm 0.71$ ) using the EMP primer set (1391F/EukBr), corresponding to the amplification efficiency of $80-87 \%$. 1183F/1631R and 563F/1132R exhibited similar PCR efficiencies, whereas $616 * \mathrm{~F} / 1132 \mathrm{R}$ showed lower efficiency (Supplementary Fig. S1A). To assess the avoidance of bacterial DNA amplification, we used a bacterial DNA mixture. Amplification from $0.1 \mathrm{ng}$ of bacterial DNA (final concentration of $0.01 \mathrm{ng} / \mu \mathrm{l}$ ), which corresponds to $\sim 20000$ copies of rRNA, required $\sim 27$ cycles (mean $\mathrm{Ct} \pm \mathrm{SD}=27.10 \pm 0.81)$ using the EMP primer set. Ct difference between eukaryotic and bacterial DNA was the largest for $1183 \mathrm{~F} / 1631 \mathrm{R}$, followed by $616^{*} \mathrm{~F} / 1132 \mathrm{R}$, whereas this difference was the smallest for the EMP primer set. The results for $28 \mathrm{~S}$ rDNA primer sets are shown in Supplementary Fig. S1B. Amplification efficiencies of the 28 S primer sets for C. elegans DNA exceeded 70\% except for that of RM3F/RM4R, which required 12-16 additional cycles for amplification. All $28 \mathrm{~S}$ primers demonstrated lower sensitivity to bacterial DNA and required more than 10 additional PCR cycles compared with the EMP primer set.

The detection limits of C. elegans DNA using the primer sets were $0.2-2 \mathrm{pg}$, corresponding to 1-10 C. elegans cells. Products were detected for no-template negative controls at approximately 30 cycles using RM2F/RM3R compared with approximately 35 cycles using the EMP primer sets. No non-specific amplification was detected with 40 cycles using the other primer sets.

Based on these results, we selected one primer set for each variable region of $28 \mathrm{~S}$ rDNA, namely DM568F/ RM2R for D3-4, RM2F/RM3R for D4-5, GA12F/RM4R for D5-6 and GA20F/RM9R for D8-9.

Deep sequencing. Next, we performed MiSeq analysis of $18 \mathrm{~S}$ or $28 \mathrm{~S}$ rDNA amplicons using the two conventional and six newly selected primer sets. We used DNA extracted from the faeces of wild rats and a domesticated bovid as templates, which were anticipated to be highly rich in bacteria. Our previous morphological observations have revealed that five rats (i.e., WR4-8) were heavily infected with parasitic nematodes, while one rat (ZR4) was infected with tapeworms ${ }^{4}$. In contrast, the bovine sample (MB1) was rich in protozoan parasites. In total, 1,311,788 high-quality reads, with a mean of 23,425 reads per test (samples $\times$ primers), were obtained via Illumina MiSeq (Table S4).

Taxonomic classification of the sequence reads revealed that EMP primer set (1391F/EukBr) amplicons contained numerous bacterial reads, with the highest observed in ZR4 (approximately 53\%) and the lowest in WR5 (approximately 10\%) (Fig. 1; Supplementary Table S5). 563F/1132R amplicons contained more bacterial reads than the EMP primer set amplicons for all samples. In particular, MB1 contained approximately $97 \%$ bacterial reads. $616 \mathrm{~F}^{*} / 1132 \mathrm{R}$ amplicons contained fewer bacterial reads $(<10 \%)$ than EMP primer set amplicons for all samples. The other primer set amplicons (i.e. 1183F/1631R, DM568F/RM2R, RM2F/RM3R, GA12F/RM4R and GA20F/RM9R) contained none or only a few bacterial reads $(0-0.15 \%)$.

Archaea reads were detected in EMP, 616*F/1132R and GA20/RM9R amplicons, although few ( $<5 \%)$, except for MB1 amplicons of the EMP primer set which contained $>30 \%$ Archaea reads. Relatively more unassigned 


\section{Level 7}
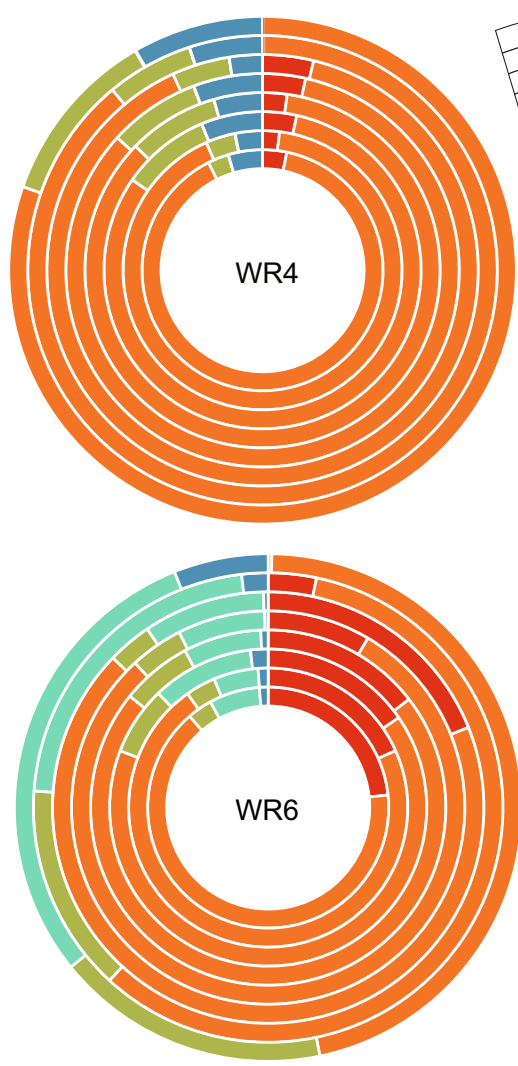

\section{Taxonomy (Level7)}

- Opisthokonta;Holozoa;Metazoa (Animalia);Eumetazoa;Bilateria;Chordata

- Opisthokonta;Holozoa;Metazoa (Animalia);Eumetazoa;Bilateria;Nematoda

- Opisthokonta;Nucletmycea;Fungi;Dikarya;Ascomycota;Saccharomycoina

- SAR;Alveolata;Apicomplexa;Conoidasida;Coccidia;Eimeriorina

Others

\section{Level 10}
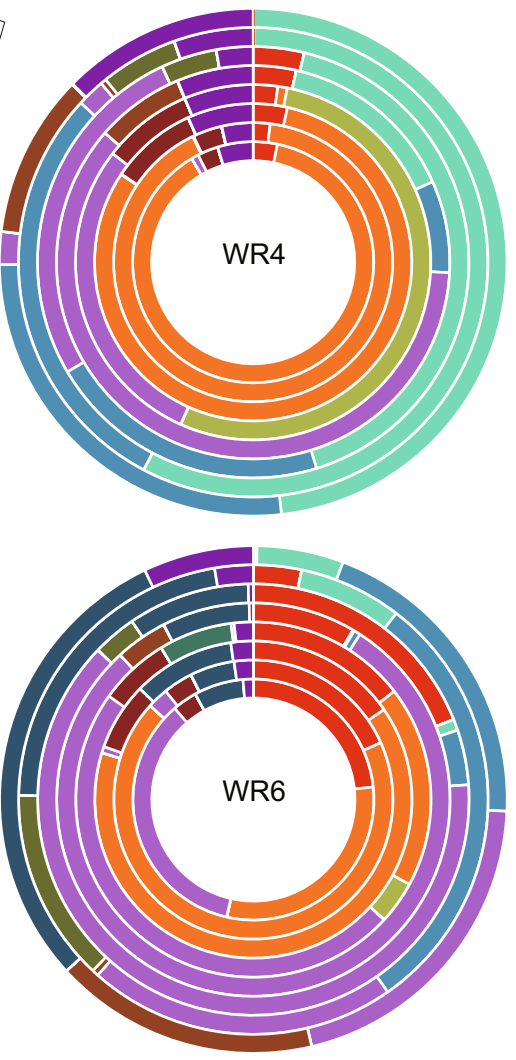

Taxonomy (Level10)

- Chordata;Vertebrata;Gnathostomata;Euteleostomi

- Nematoda;Chromadorea;Rhabditida;Ambiguous_taxa

- Nematoda;Chromadorea;Rhabditida;Ancylostoma

- Nematoda;Chromadorea;Rhabditida;Heligmosomoides

- Nematoda;Chromadorea;Rhabditida;Nippostrongylus

- Nematoda;Chromadorea;Rhabditida;Strongyloides

- Saccharomycotina;Saccharomycetes;Saccharomycetales;Ambiguous_taxa - Saccharomycotina;Saccharomycetes;Saccharomycetales;Kazachstania

- Saccharomycotina;Saccharomycetes;Saccharomycetales;Saccharomyces

- Eimeriorina;Ambiguous_taxa

- Eimeriorina;Eimeria

Others

Figure 2. Taxonomic classification of eukaryotic reads in the faecal samples of nematode-infected rats (WR4 and WR6) at SILVA levels 7 and 10. Circles from the inside show the taxonomic distributions of reads obtained using the EMP (1391F/EukBr), 563F/1132R, 616*F/1132R, 1183F/1631R, DM568F/RM2F, RM2F/RM3R, GA12F/RM4R and GA20F/RM9R primer sets, respectively. Only taxa with $\geq 5 \%$ of the total non-bacterial reads are shown in the plots, and taxa with $<5 \%$ are summarised as 'Others'. Sequence reads without any taxonomic assignments because of low similarity with the database are shown as 'Unassigned'.

reads (displaying no similarity with sequences in the database) were detected in MB1 amplicons of EMP, $616 * \mathrm{~F} / 1132 \mathrm{R}$ and $1183 \mathrm{~F} / 1631 \mathrm{R}$ primer sets $(8.5 \%, 9.0 \%$ and $10.7 \%$, respectively). Other combinations of primer sets and DNA samples revealed few unassigned reads $(<2.2 \%)$.

Numbers of operational taxonomic units (OTUs) detected using the EMP primer set ranged from 150 to 400 (Table S4). However, approximately half of the OTUs were assigned to either Bacteria or Archaea. Although the number of OTUs detected using 563F/1132R was the highest for each DNA sample ( $>1200$ OTUs), after removing the bacteria and archaea reads, this number became the lowest among all primer sets. Other primer sets detected few or no bacterial OTUs, and the eukaryotic OTUs ranged from 40 to 500. Among these, RM2F/RM3R detected the lowest number of OTUs in the six rat samples, whereas GA12/RM4R detected the lowest numbers in the bovine sample. Overall, the six newly selected primer sets more readily avoided bacterial DNA amplification than the conventional primer sets. Finer classifications after removing the bacteria and archaea reads are shown in Figs 2-4. 


\section{Level 7}

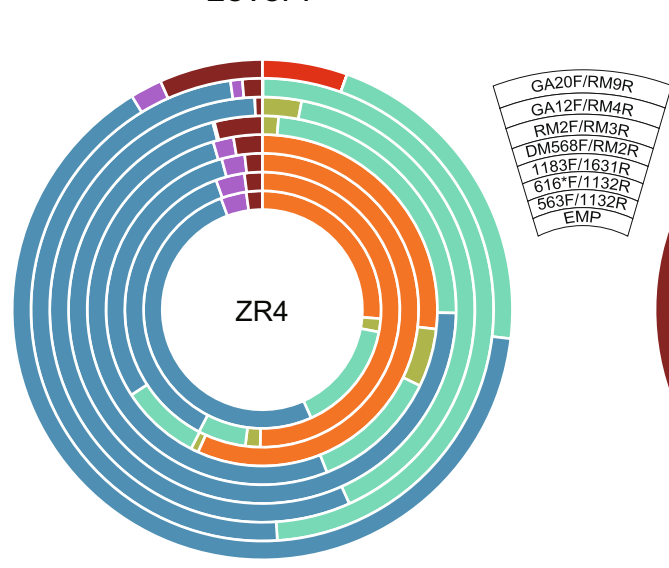

Level 10

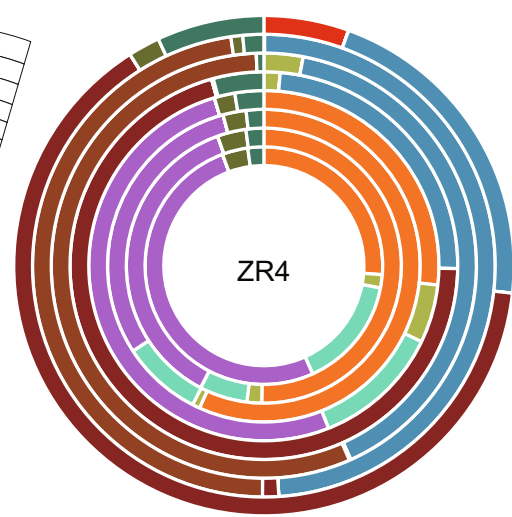

\section{Taxonomy (Level7)}

- Excavata;Metamonada;Parabasalia;Mastotermes

- Excavata;Metamonada;Parabasalia;Tritrichomonadea;Tritrichomonas

- Opisthokonta;Holozoa;Metazoa (Animalia);Eumetazoa;Bilateria;Chordata

- Opisthokonta;Holozoa;Metazoa (Animalia);Eumetazoa;Bilateria;Platyhelmin

- Opisthokonta;Nucletmycea;Fungi;Dikarya;Ascomycota;Saccharomycotina

- Unassigned

- Others

\author{
Taxonomy (Level10) \\ - Parabasalia;Mastotermes \\ - Parabasalia;Tritrichomonadea;Tritrichomonas \\ - Chordata;Vertebrata;Gnathostomata;Euteleostomi \\ $=$ Platyhelminthes;Cestoda;Eucestoda;Cyclophyllidea \\ - Platyhelminthes;Cestoda;Eucestoda;Hymenolepis \\ - Saccharomycotina;Saccharomycetes;Saccharomycetales;Ambiguous_taxa \\ - Saccharomycotina;Saccharomycetes;Saccharomycetales;Kazachstania \\ - Saccharomycotina;Saccharomycetes;Saccharomycetales;Saccharomyces \\ - Unassigned \\ - Others
}

Figure 3. Taxonomic classification of eukaryotic reads in the faecal samples of a tapeworm-infected rat (ZR4) at SILVA levels 7 and 10. Circles from the inside show taxonomic distributions using the EMP (1391F/EukBr), 563F/1132R, 616*F/1132R, 1183F/1631R, DM568F/RM2F, RM2F/RM3R, GA12F/RM4R and GA20F/RM9R primer sets, respectively. Only taxa with $\geq 5 \%$ of the total non-bacterial reads are shown in the plots, and taxa with $<5 \%$ are summarised as 'Others'. Sequence reads without any taxonomic assignments because of low similarity with the database are shown as 'Unassigned'.

Nematode-infected samples. At the phylum level (SILVA level 7) classification, all primer sets exhibited similar taxon distribution patterns in WR4, although small proportional differences were noted (Fig. 2). Many reads (46-91\%) were assigned to the phylum Nematoda and some were assigned to the phylum Chordata and sub-phylum Saccharomycotina using all primer sets. At SILVA level 10, Nematoda reads were further classified to the family or genus level. Using the three $18 \mathrm{~S}$ primer sets (i.e. EMP, 563F/1132R and 616*F/1132R), many (>85\%) Nematoda reads was assigned to 'Rhabditida; Ambiguous'. Conversely, using 1183F/1631R, the proportion of ambiguous taxa became smaller and more reads were assigned to genera such as Strongyloides and Ancylostoma. Using the $28 \mathrm{~S}$ primer sets, no 'Rhabditida; Ambiguous' reads were detected and all Nematoda reads were subdivided into genera, including Heligmosomoides, Nippostrongylus and Strongyloides; this trend was similar to the nematode taxon distribution observed in our previous morphological identification ${ }^{4}$. Similar results were noted in other WR samples (i.e. WR5, WR7 and WR8), although differences in minor taxon distributions were observed (Supplementary Tables S7 and S8). In WR6, Eimeriorina was detected in addition to Nematoda, Chordata and Saccharomycotina using all primer sets (Fig. 2). At SILVA level 10, the Eimeriorina reads were further classified into Eimeria or 'Eimeriorina; Ambiguous' taxa.

Tapeworm-infected samples. ZR4 harboured Hymenolepis tapeworms in its intestine. At SILVA level 7, all $18 \mathrm{~S}$ primer sets detected high proportions of Platyhelminthes as well as Saccharomycotina and Trichomonas (or 'Trichomonas; Ambiguous') (Fig. 3; Supplementary Table S7). The three 28S primer sets did not detect Trichomonas reads, while GA20F/RM9R detected few Trichomonas reads (approximately 0.1\%); therefore, Saccharomycotina and Platyhelminthes occupied higher proportions of the total reads using the 28S primer sets than using the $18 \mathrm{~S}$ primer sets. Chordata reads were detected by all $18 \mathrm{~S}$ primers and two $28 \mathrm{~S}$ primer sets (i.e., DM568F/RM2R and RM2F/RM3R). Mastotermes was detected only by the GA20F/RM9R primer set. At SILVA level 10, Platyhelminthes reads were further classified to the order Cyclophyllidea using the 18S primer sets and to the genus Hymenolepis using the $28 \mathrm{~S}$ primer sets. Saccharomycotina was further classified to the order Saccharomycetales using the $18 \mathrm{~S}$ primer sets and to the genera Saccharomyces and Kazachstania using the $28 \mathrm{~S}$ primer sets.

Protozoa-rich samples. Various protozoa occur in the bovine gastrointestinal tract; thus, protozoal cysts are frequently detected in faecal samples. Most of these protozoa form a part of the normal ruminal microflora called ciliated protozoa ${ }^{25,26}$; however, some of these, such as Eimeria (Coccidia), Cryptosporidium, Giardia, 
Level 4

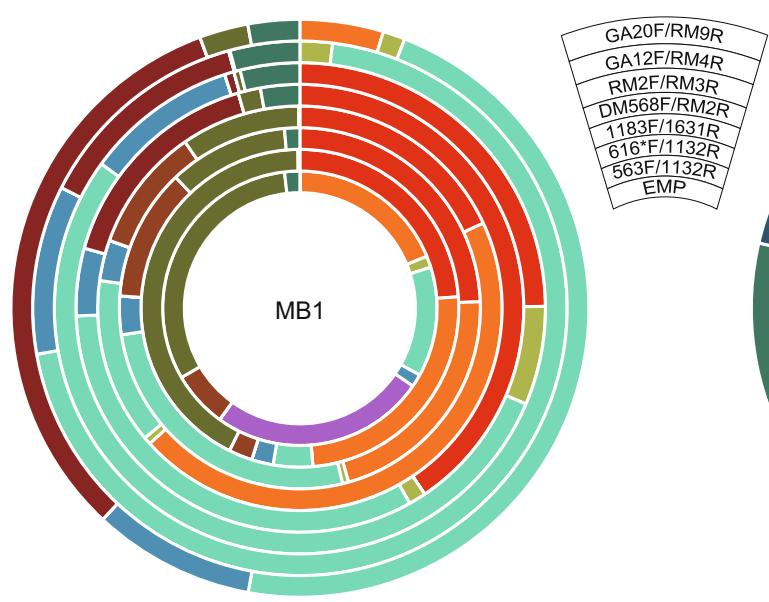

Level 7

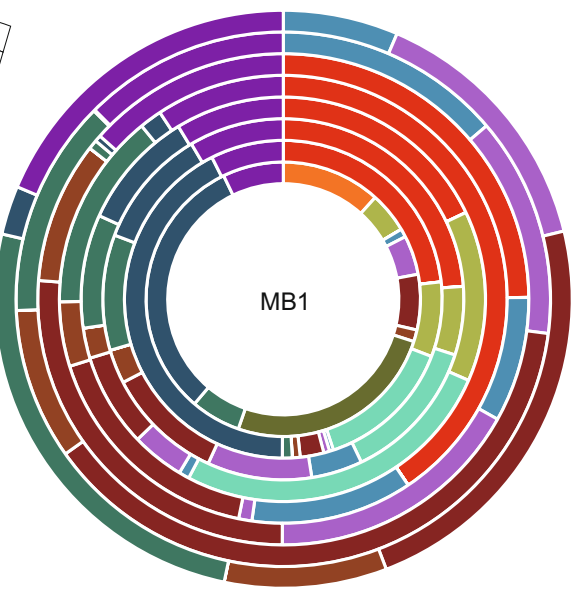

Taxonomy (Level4)

- Amoebozoa;Archamoebae;Entamoebida
- Excavata;Metamonada;Parabasalia
- Opisthokonta;Holozoa;Metazoa (Animalia)
- Opisthokonta;Nucletmycea;Fungi
- SAR;Alveolata;Apicomplexa
- SAR;Rhizaria;Retaria
- SAR;Stramenopiles;Blastocystis
- SAR;Stramenopiles;Incertae Sedis
- Unassigned
- Others

Taxonomy (Level7)

\author{
- Entamoebida;Entamoeba;Entamoeba \\ - Parabasalia;Trichomonadea;Ditrichomonas;Ditrichomonas \\ - Parabasalia;Trichomonadea;Pentatrichomonas;Pentatrichomonas \\ - Parabasalia;Trichomonadea;Simplicimonas \\ - Fungi;Dikarya;Ascomycota;Pezizomycotina \\ - Fungi;Dikarya;Ascomycota;Saccharomycotina \\ - Fungi;Neocallimastigomycota;Incertae Sedis;Neocallimastigomycetes \\ - Apicomplexa;Conoidasida;Coccidia;Eimeriorina \\ - Retaria;RAD B;Ambiguous_taxa \\ - Blastocystis;Blastocystis \\ - Unassigned \\ - Others
}

Figure 4. Taxonomic classification of eukaryotic reads in protozoa-rich bovine faecal samples (MB1) at SILVA levels 4 and 7. Circles from the inside show taxonomic distributions using the EMP (1391F/EukBr), 563F/1132R, 616*F/1132R, 1183F/1631R, DM568F/RM2F, RM2F/RM3R, GA12F/RM4R and GA20F/RM9R primer sets, respectively. Only taxa having $\geq 5 \%$ of the total non-bacterial reads are shown in the plots, and taxa with $<5 \%$ are summarised in 'Others'. Sequence reads without taxonomic assignments because of low similarity with the database are shown as 'Unassigned'.

Entamoeba and Trichomonas, are pathogenic and thus possess clinical significance ${ }^{27}$. In this study, we used bovine faeces as prototypical protozoa-rich samples.

At SILVA level 4, the EMP primer set (1391F/EukBr) detected reads assigned in descending order to Retaria, Parabasalia, Fungi, Stramenopiles, Apicomplexa and Metazoa (Fig. 4; Supplementary Table S6). Concomitantly, approximately $30 \%$ of the reads did not share similarities with known rDNA sequences in the database (unassigned). The other $18 \mathrm{~S}$ primer sets produced similar patterns as the EMP primer sets. Approximately $10-40 \%$ of the reads were unassigned, and the remaining reads were primarily assigned to Parabasalia, Fungi, Apicomplexa and Stramenopiles. The largest differences from the EMP primer sets were Retaria and Entamoebida, which were detected only by the EMP and only by the other three $18 \mathrm{~S}$ primer sets, respectively. The $28 \mathrm{~S}$ primer sets detected lower proportions of unassigned reads than the $18 \mathrm{~S}$ primer sets. The main detected taxa were similar between the $28 \mathrm{~S}$ and $18 \mathrm{~S}$ primer sets. However, Parabasalia reads were not detected by DM568F/RM2R, RM2F/RM3R and GA12F/RM4R, whereas Entamoebida reads were not detected by GA12F/RM4R and GA20F/RM9R. Instead of 'Stramenopiles; Incertae sedis', four $28 \mathrm{~S}$ primer sets detected Stramenopiles; Blastocystis, although the proportion with RM2F/RM3R was minute.

At SILVA level 7, Parabasalia detected by the $18 \mathrm{~S}$ primer sets were further classified into Trichomonadea taxa, including Trichomonas, Ditrichomonas, Tetratrichomonas, Pentatrichomonas and Simplicimonas (Fig. 4; Supplementary Table S7). Apicomplexa were further classified to Eimeriorina using all primer sets. Fungi were subdivided into the orders Neocallimastigomycetena, Saccharomycotina and Pezizomycotina, albeit without noticeable differences among the primer sets.

Beta diversity analyses. A technical replicate experiment was performed from PCR amplification to MiSeq independently from the first experiment using the newly selected primer sets (Dataset 2; Supplementary Tables S9-S12). The dendrograms of cluster analysis based on the Bray-Curtis dissimilarity of taxon abundance 
A

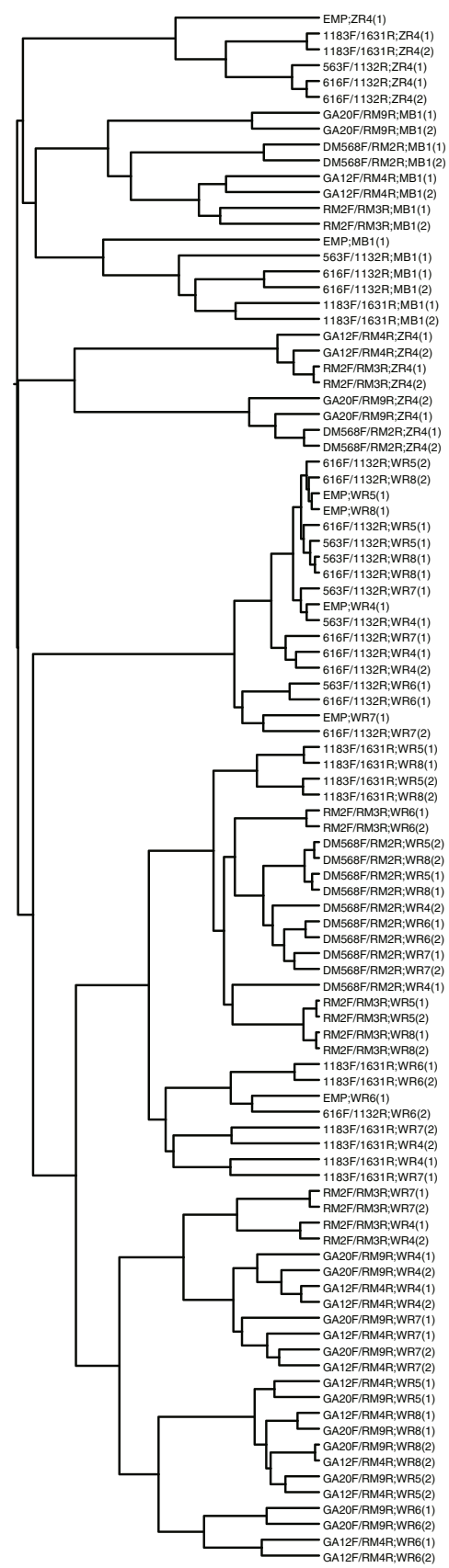

$\overline{0.05}$
B

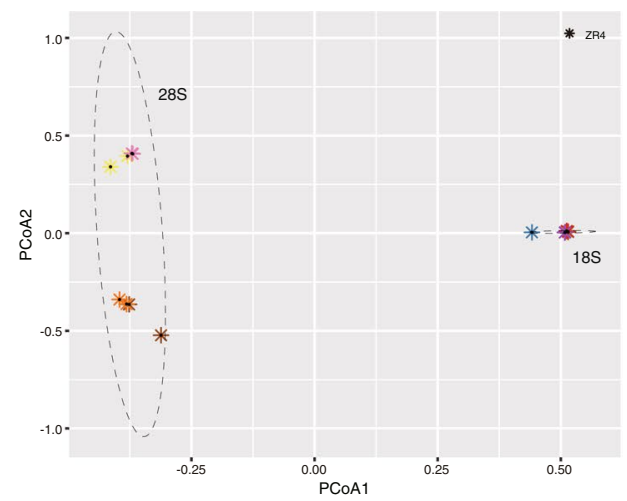

C

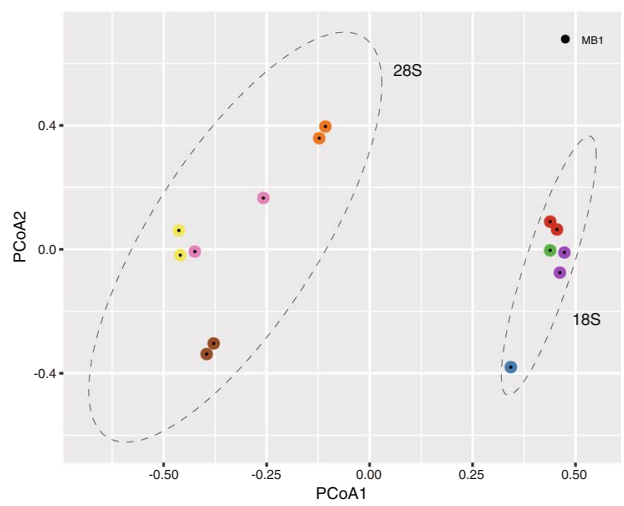

D

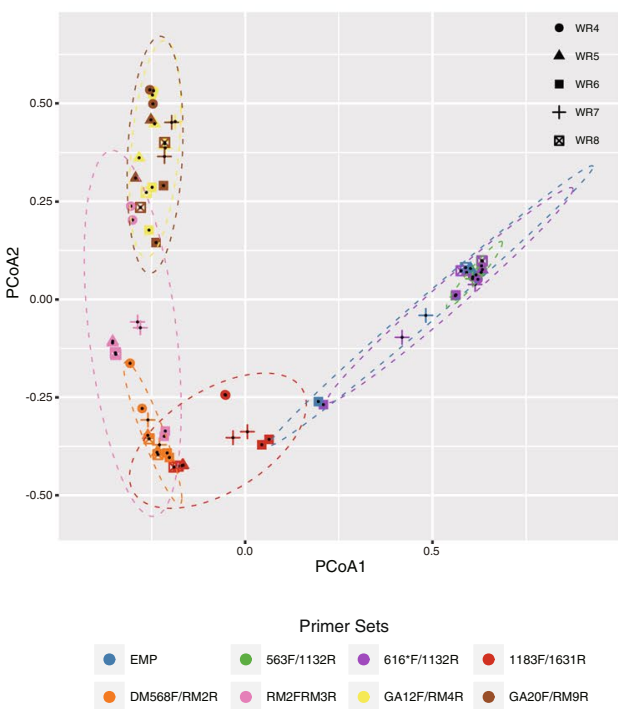

Figure 5. Cluster analysis and principal coordinate analysis (PCoA) of relative taxon abundance. (A) Dendrogram of cluster analysis based on the Bray-Curtis similarity index for the two replicate datasets. The labels on the edges represent 'primer sets; DNA samples (dataset)'. (B-D) PCoA plots of ZR4, MB1 and WR samples, respectively. Ellipses drawn around the dots represent the 95\% confidence limit for PCoA1 and 2 for $18 \mathrm{~S}$ and $28 \mathrm{~S}$ groups $(\mathbf{B}, \mathbf{C})$ or primer sets $(\mathbf{D})$.

from the two replicate experiments are shown in Fig. 5A. All replicates (Datasets 1-2) in MB1 and ZR4 were clustered together in the dendrogram, suggesting high reproducibility of the methods using these primer sets (Fig. 5A). For WR samples, although the replicates were largely clustered together, some technical replicates were nested within the other DNA samples (e.g. WR4 with WR7 and WR5 with WR8), perhaps because those samples showed very similar taxonomic compositions. Principal coordinates analysis (PCoA) plots were generated for 
ZR4, MB1 and WR samples (Fig. 5B-D, respectively). In the three plots, PCoA1 separated the samples based on the PCR target regions (18S or $28 \mathrm{~S}$ ), although the separation in the WR plot, which contained five DNA samples, was not as obvious as that in the other plots. Among the $28 \mathrm{~S}$ primer sets, RM2F/RM3R and GA12F/RM4R were clustered together in all the plots, whereas DM568F/RM2R and GA20F/RM4R were clustered together in the ZR and WR plots but not in the MB plot. Among the $18 \mathrm{~S}$ primer sets, $563 \mathrm{~F} / 1132 \mathrm{R}$ and $616^{*} / \mathrm{F} 1132 \mathrm{R}$ were clustered together in all the plots. These results correspond to the target regions of $18 \mathrm{~S}$ or $28 \mathrm{~S}$ (Tables 1 and 2 ).

\section{Discussion}

Bacterial read contamination of PCR amplicons often poses a critical problem in NGS-based analyses of eukaryotic diversity or diagnoses. In extreme cases, as with MiSeq of a bovine faecal sample in the present study, over $95 \%$ of the total sequence reads can be derived from bacterial DNA, making it difficult to detect rare eukaryotes in the samples. Increasing data acquisition may resolve this issue; however, presence of raw data with only one or two orders of magnitude than non-contaminated cases is inefficient and therefore prevents high-throughput analyses. The primer sets newly screened in this study, which can efficiently amplify rDNA from a wide range of eukaryotes without bacterial DNA amplification, are anticipated to be suitable tools for diversity analyses of eukaryotic microbes, including parasites.

At the same time, we noted that each primer set could not detect a specific taxonomy groups. According to our deep sequencing analysis, two of the $28 \mathrm{~S}$ primer sets could not to detect Trichomonas species, which were detected by all other $18 \mathrm{~S}$ primer sets. Spironucleus reads were detected from rat faeces using only one $28 \mathrm{~S}$ primer set (GA20F/RM9R). Entamoeba could not be detected using the EMP and GA20F/RM9R primer sets. In addition, the results of in silico analysis suggested that one primer set is unlikely to cover all taxonomic groups of parasites. For instances, Plasmodium spp., one of the most medically important parasites, was difficult to detect using any of the tested $18 \mathrm{~S}$ rDNA primer sets, although it may be detected using the two 28S rDNA primer sets. Trypanosoma and Leishmania, two other important parasitic genera, could be detected only using 563F/1132R and $1183 \mathrm{~F} / 1631 \mathrm{R}$ among the tested primers. Collectively, these results suggest the importance of selecting primer sets according to the study objective.

To achieve fine taxonomic resolution, long sequences containing sufficient diversity to distinguish closely related species are essential. Although sequencing technologies capable of producing long sequences, such as PacBio and NanoPore, are available ${ }^{28,29}$, these remain impractical for rDNA-based microbiome analyses because of their higher error rates and lower throughputs than those of Illumina sequencing. Therefore, many studies have used Illumina sequencing, for which the maximum length is $600 \mathrm{bp}$ (300-bp paired-end). Although we used variable regions of $18 \mathrm{~S}$ rDNA with fragment lengths ranging from 150 to 570 for taxonomic classification, we were unable to further assign the reads to the genus or species level in most cases. On the contrary, reads of $28 \mathrm{~S}$ rDNA, which has higher sequence diversity than $18 \mathrm{SDNA}^{13}$, could sometimes be further assigned to the genus level, suggesting that $28 \mathrm{~S}$ rDNA represents a good option for studies in which finer classification is necessary. One of the challenges in $28 \mathrm{~S}$ rDNA-based population analyses is the enlargement of the database because database sizes affect fine taxonomic classification. The current database (SILVA r132) contains 198,843 28S rDNA sequences compared with 695,171 18S rDNA sequences (https://www.arb-silva.de/). In addition, we discarded primer sets with amplicon sizes that were out of range even though they demonstrated good taxonomic coverages (Supplementary Table S13). These primers can be used as alternates if they are capable of amplifying sequences to meet the length requirement.

Host DNA contamination did not hamper analyses in this study. Small proportions of mammalian (Chordata) reads were detected with any combination of samples and primers. This is probably because the faecal samples used in this study were collected from wild animal and contained high number of eukaryotic microbes. However, our in-silico analysis revealed that all the tested primer sets theoretically cannot avoid amplification of host DNA. Therefore, when samples are expected to have small amounts of eukaryotic microbes, such as clinical samples from human or samples from well-kept pets, PCR blockers may be required, which prevent host DNA amplification $^{30-32}$. Applying taxon-specific primers is an alternative option to avoid amplification of host DNA. Recently, Cannon et al..$^{33}$ proposed a high-throughput method to detect a wide range of parasites by a combination of multiple taxon-specific primers. We tested those primers using our evaluation criteria and confirmed that those primer sets amplify each targeted taxa and can avoid host and bacterial DNA amplification (Table S14). Although this strategy requires optimisation for multiplex PCR (amplification of multiple targets in a single PCR) for high throughput studies and may require a reasonable normalisation method for amplification bias by each primer set for a reliable estimation of taxa distribution in a sample, the assay still has an advantages in customizability to easily include additional targeted taxa ${ }^{33}$. Therefore, the primer sets selected in this study can be added to the multiplex assay, which could achieve more comprehensive "parasitome" analyses.

The benefits and drawbacks of the newly selected primer sets and conventional primers are summarised in Table 3. First, the newly selected primer sets could avoid bacterial DNA amplification. However, taxonomic coverage differed with each primer set. Ultimately, the primer sets should be selected according to the study objectives, taking the parasites that need to be covered and the required resolution into account. However, we recommend the use of $616 * / F 1132 \mathrm{R}$ for $18 \mathrm{~S} \mathrm{rDNA}$ or DM568F/RM2R for $28 \mathrm{~S} \mathrm{rDNA}$, or a combination of those, as new standard primer sets for parasite detection because these provide wide taxonomic coverage of parasitic eukaryotes with minimal bacterial DNA contamination.

\section{Methods}

SSU and LSU primer screening. Potential universal primer sequences targeting eukaryote rDNA were obtained from previous studies $5,6,13,23,24$. The primers were filtered to select primer pairs suitable for Illumina MiSeq analysis under the following criteria: Tm in the range of $55^{\circ} \mathrm{C}-70^{\circ} \mathrm{C}$, a difference in $\mathrm{Tm}$ between the two primers of $<5^{\circ} \mathrm{C}$ and an amplicon size of $200-580 \mathrm{bp}$. These primer pairs were further evaluated for similarities 


\begin{tabular}{|c|c|c|c|c|c|c|c|c|}
\hline & $\begin{array}{l}\text { EMP (1391F/ } \\
\text { EukBr) }\end{array}$ & $563 F / 1132 R$ & $616 * \mathrm{~F} / 1132 \mathrm{R}$ & $1183 \mathrm{~F} / 1631 \mathrm{R}$ & $\begin{array}{l}\text { DM568F/ } \\
\text { RM2R }\end{array}$ & $\begin{array}{l}\text { RM2F/ } \\
\text { RM3R }\end{array}$ & $\begin{array}{l}\text { GA12F/ } \\
\text { RM4R }\end{array}$ & $\begin{array}{l}\text { GA20F/ } \\
\text { RM9R }\end{array}$ \\
\hline rDNA & $18 \mathrm{~S}$ & $18 \mathrm{~S}$ & $18 \mathrm{~S}$ & $18 \mathrm{~S}$ & $28 \mathrm{~S}$ & $28 \mathrm{~S}$ & $28 \mathrm{~S}$ & $28 \mathrm{~S}$ \\
\hline Target variable region & V9 & V4-5 & V4-5 & V7-8 & D4-5 & D4-5 & D4-6 & D8-9 \\
\hline Degeneracy (forward/reverse) & $0 / 0$ & $3 / 3$ & $4 / 3$ & $0 / 0$ & $0 / 1$ & $1 / 1$ & $0 / 5$ & $1 / 1$ \\
\hline Amplicon size (bp) $)^{\mathrm{a}}$ & 145 & 569 & 516 & 449 & 284 & 236 & 507 & 505 \\
\hline Bacterial contamination & - & -- & + & ++ & ++ & ++ & ++ & ++ \\
\hline \multicolumn{9}{|l|}{ Taxonomic coverage ${ }^{b}$} \\
\hline Nematoda & + & ++ & ++ & ++ & + & ++ & ++ & ++ \\
\hline Platyhelminthes & + & ++ & ++ & ++ & + & ++ & ++ & ++ \\
\hline Acanthocephala & + & ++ & ++ & - & ++ & ++ & ++ & ++ \\
\hline Coccidia & + & ++ & ++ & ++ & ++ & ++ & ++ & ++ \\
\hline Cryptosporida & + & ++ & ++ & ++ & ++ & ++ & ++ & ++ \\
\hline Haemosporidia & + & - & - & - & ++ & ++ & - & ++ \\
\hline Fornicata & + & ++ & + & + & + & - & - & ++ \\
\hline Discicristata & + & ++ & + & ++ & + & + & ++ & + \\
\hline Parabasalia & + & ++ & + & - & ++ & - & + & ++ \\
\hline Entamoebida & + & ++ & ++ & - & ++ & - & - & - \\
\hline Longamoebia & + & ++ & ++ & ++ & ++ & ++ & ++ & ++ \\
\hline
\end{tabular}

Table 3. A summary of $18 \mathrm{~S}$ and $28 \mathrm{~S}$ rDNA primer set evaluation. ${ }^{\mathrm{a} B a s e d}$ on $\mathrm{S}$. cerevisiae rRNA gene (NC_001144). ${ }^{\mathrm{b}}++;>85 \%,+; 10-85 \%,-;<10 \%$ in the in silico taxonomy coverage test. Bold characters indicate detections confirmed using MiSeq sequencing in this study.

with eukaryote and bacterial rDNA sequences using TestPrime 1.0 and the SILVA 132 database under the following parameters: maximum number of mismatch $=4 \mathrm{bp}$ and the length of 0 -mismatch zone at the $3^{\prime} \mathrm{end}=3 \mathrm{bp}$ ). We used the non-redundant reference dataset (Ref NR) build by a dereplication of the full reference set using a $99 \%$ identity criterion and were suggested by SILVA to be used as a representative dataset for classification, phylogenetic analysis and probe design.

DNA samples. A bacterial DNA mixture was prepared by combining $70 \mathrm{ng}$ DNA extracted from pure cultures of seven bacterial species (Escherichia coli, Enterobacter sp., Serratia sp., Bacillus subtilis, Klebsiella pneumoniae, Group A Streptococcus and Staphylococcus epidermidis) using a QIAmp DNA Mini Kit (Qiagen). C. elegans DNA was extracted from approximately 10,000 worms using the same kit.

For MiSeq analyses, DNA extracted from the faeces of rats caught in the Miyazaki City Phoenix Zoo (ZR, Rattus rattus) or in Miyazaki downtown (WR, Rattus norvegicus) in our previous study ${ }^{4}$ were used. Faecal samples from a domesticated bovid (MB, Bos taurus) were provided by the veterinary parasitology lab of the University of Miyazaki, and DNA was extracted using a Maxwell RSC Purefood GMO Kit (Promega), as described previously ${ }^{34}$.

qPCR. qPCR was performed to test the amplification efficiency of each primer set using C. elegans DNA or the bacterial DNA mixture as a template. Reactions were performed in triplicates using a StepOnePlus Real-Time PCR System (Applied Biosystems) under the following conditions: $95^{\circ} \mathrm{C}$ for $10 \mathrm{~min}$, followed by 40 cycles of $95^{\circ} \mathrm{C}$ for $15 \mathrm{~s}, 50^{\circ} \mathrm{C}$ for $30 \mathrm{~s}$ and $60^{\circ} \mathrm{C}$ for $1 \mathrm{~min}$ (for $18 \mathrm{~S} \mathrm{rDNA}$ amplification), or $95^{\circ} \mathrm{C}$ for $10 \mathrm{~min}$, followed by 40 cycles of $95^{\circ} \mathrm{C}$ for $15 \mathrm{~s}$ and $60^{\circ} \mathrm{C}$ for $1 \mathrm{~min}$ (for $28 \mathrm{~S} \mathrm{rDNA}$ amplification). The reaction volume was $10 \mu \mathrm{l}$, including $5 \mu \mathrm{l}$ of the Power SYBR Green PCR Master Mix $(2 \mathrm{x}), 0.9 \mu \mathrm{M}$ of each primer and $1 \mu \mathrm{l}$ of DNA solution. To calculate the PCR efficiencies and detection limits, serial 10-fold dilutions of C. elegans DNA ( $1 \mathrm{ng}$ to $0.01 \mathrm{pg}$ ) were used as templates.

MiSeq sequencing. PCR was performed using Tks Gflex DNA Polymerase (Takara), and a 30- $\mu$ l reaction mixture containing $1 \mu \mathrm{l}$ of template DNA (1-3 ng of DNA), $15 \mu \mathrm{l}$ of $2 \times$ Gflex buffer, $0.5 \mu \mathrm{l}$ each of the forward/ reverse primers with the Illumina MiSeq Adapter ( $10 \mu \mathrm{M}$ final concentration), $0.5 \mu \mathrm{l}(100 \mathrm{U})$ of DNA polymerase and $13 \mu \mathrm{l}$ of nuclease-free $\mathrm{H}_{2} \mathrm{O}$. Reactions were performed using Veriti Thermal Cycler (Applied Biosystems) under the following conditions: $95^{\circ} \mathrm{C}$ for $1 \mathrm{~min}$, followed by 35 cycles of $95^{\circ} \mathrm{C}$ for $15 \mathrm{~s}, 60^{\circ} \mathrm{C}$ (for $28 \mathrm{~S}$ rDNA amplification) or $50^{\circ} \mathrm{C}\left(18 \mathrm{~S}\right.$ rDNA amplification) for $1 \mathrm{~min}$ and $68^{\circ} \mathrm{C}$ for $1 \mathrm{~min}$. Duplicate PCRs were performed independently, and the produced materials were then mixed. The PCR products were confirmed via agarose gel electrophoresis and purified using AMpure XP beads (Beckman Coulter). Index PCR was performed to attach dual indices and Illumina sequencing adapters to the first PCR products using the Nextera XT Index Kit (Illumina) and KAPA HiFi HotStart Ready Mix (Kapa Biosystems) under the following conditions: $95^{\circ} \mathrm{C}$ for $3 \mathrm{~min}$, followed by $8 \mathrm{cycles}$ of $95^{\circ} \mathrm{C}$ for $30 \mathrm{~s}, 55^{\circ} \mathrm{C}$ for $30 \mathrm{~s}$ and $72^{\circ} \mathrm{C}$ for $30 \mathrm{~s}$, and the final extension at $72^{\circ} \mathrm{C}$ for $5 \mathrm{~min}$. The PCR product was cleaned using AMpure XP beads, pooled at equal concentrations and then sequenced using the MiSeq Reagent Nano Kit v3 (600 cycles) according to the manufacturer's protocol (http:// icom.illumina.com/) to produce 300 -bp paired-end reads.

Bioinformatic analysis. Illumina sequence data were processed using QIIME version 1.9.135. Paired-end reads were joined using the 'fastq-join' method (join_paired_ends.py). After QIIME quality filtering (split_libraries_ fastq.py: -store_qual_scores -q 9 -max_barcode_errors 2 -sequence_max_n 1 -max_bad_run_length 2 -p 0.5 -r 3), 
chimeric sequences were detected using the UCHIME algorithm, which is included in the free version of USEARCH61, and eliminated from further analyses. Cleaned reads were clustered and assigned to OTUs using the open-reference OTU-picking protocol with the SILVA 128 database ${ }^{36}$ at $97 \%$ identity with 'blast' (pick_open_ reference_otus.py).

Similarity in taxa composition and the relative abundance were analysed via PCoA and hierarchical cluster analyses using the Bray-Curtis similarity index with $\mathrm{R}$ vegan package $\mathrm{e}^{37}$.

\section{Data availability}

The sequencing data have been deposited to the DNA Data Bank of Japan Sequence Read Archive under the BioProject PRJDB3050.

Received: 4 February 2019; Accepted: 7 October 2019;

Published online: 31 October 2019

\section{References}

1. Langille, M. G. et al. Predictive functional profiling of microbial communities using 16S rRNA marker gene sequences. Nature biotechnology 31, 814 (2013).

2. Turnbaugh, P. J. et al. The effect of diet on the human gut microbiome: a metagenomic analysis in humanized gnotobiotic mice. Science translational medicine 1, 6ra14, https://doi.org/10.1126/scitranslmed.3000322 (2009).

3. Hino, A., Maruyama, H. \& Kikuchi, T. A novel method to assess the biodiversity of parasites using $18 \mathrm{~S}$ rDNA Illumina sequencing; parasitome analysis method. Parasitology international 65, 572-575, https://doi.org/10.1016/j.parint.2016.01.009 (2016).

4. Tanaka, R. et al. Assessment of helminth biodiversity in wild rats using $18 \mathrm{~S}$ rDNA based metagenomics. PloS one 9, e110769, https:// doi.org/10.1371/journal.pone.0110769 (2014).

5. Hadziavdic, K. et al. Characterization of the $18 \mathrm{~S}$ rRNA gene for designing universal eukaryote specific primers. PloS one 9 , e87624, https://doi.org/10.1371/journal.pone.0087624 (2014).

6. Hugerth, L. W. et al. Systematic design of $18 \mathrm{~S}$ rRNA gene primers for determining eukaryotic diversity in microbial consortia. PloS one 9 , e95567 (2014).

7. Stoeck, T. et al. Multiple marker parallel tag environmental DNA sequencing reveals a highly complex eukaryotic community in marine anoxic water. Molecular ecology 19(Suppl 1), 21-31, https://doi.org/10.1111/j.1365-294X.2009.04480.x (2010).

8. Bradley, I. M., Pinto, A. J. \& Guest, J. S. Design and Evaluation of Illumina MiSeq-Compatible, 18S rRNA Gene-Specific Primers for Improved Characterization of Mixed Phototrophic Communities. Appl Environ Microbiol 82, 5878-5891, https://doi.org/10.1128/ aem.01630-16 (2016).

9. Gilbert, J. A., Jansson, J. K. \& Knight, R. Earth Microbiome Project and Global Systems Biology. mSystems 3, https://doi.org/10.1128/ mSystems.00217-17 (2018).

10. Maritz, J. M. et al. An 18S rRNA Workflow for Characterizing Protists in Sewage, with a Focus on Zoonotic Trichomonads. Microbial ecology 74, 923-936 (2017).

11. Amaral-Zettler, L. A., McCliment, E. A., Ducklow, H. W. \& Huse, S. M. A Method for Studying Protistan Diversity Using Massively Parallel Sequencing of V9 Hypervariable Regions of Small-Subunit Ribosomal RNA Genes. PLOS ONE 4, e6372, https://doi. org/10.1371/journal.pone.0006372 (2009).

12. Pawlowski, J. et al. Eukaryotic Richness in the Abyss: Insights from Pyrotag Sequencing. PLOS ONE 6, e18169, https://doi. org/10.1371/journal.pone.0018169(2011).

13. Machida, R. J. \& Knowlton, N. PCR primers for metazoan nuclear $18 \mathrm{~S}$ and $28 \mathrm{~S}$ ribosomal DNA sequences. PloS one 7, e46180, https://doi.org/10.1371/journal.pone.0046180 (2012).

14. Schoch, C. L. et al. Nuclear ribosomal internal transcribed spacer (ITS) region as a universal DNA barcode marker for Fungi. Proceedings of the National Academy of Sciences of the United States of America 109, 6241-6246, https://doi.org/10.1073/ pnas.1117018109(2012).

15. Korajkic, A. et al. Changes in bacterial and eukaryotic communities during sewage decomposition in Mississippi river water. Water research 69, 30-39 (2015).

16. Porazinska, D. L. et al. Plant diversity and density predict belowground diversity and function in an early successional alpine ecosystem. Ecology 99, 1942-1952 (2018).

17. Watts, M. P., Spurr, L. P., Gan, H. M. \& Moreau, J. W. Characterization of an autotrophic bioreactor microbial consortium degrading thiocyanate. Applied microbiology and biotechnology 101, 5889-5901 (2017).

18. Huang, T. et al. Dalangtan Playa (Qaidam Basin, NW China): Its microbial life and physicochemical characteristics and their astrobiological implications. PloS one 13, e0200949, https://doi.org/10.1371/journal.pone.0200949 (2018).

19. Benitez, E. et al. Bottom-up effects on herbivore-induced plant defences: a case study based on compositional patterns of rhizosphere microbial communities. Scientific reports 7, 6251, https://doi.org/10.1038/s41598-017-06714-x (2017).

20. Braithwaite, K. Innovative approaches to identifying the cause of chlorotic streak and new management strategies: final report 2013/357 (2017).

21. Mullins, M. T. Sample Collection and DNA Extraction Methods for Environmental DNA Metabarcoding in Headwater Streams, Eastern Kentucky University (2017).

22. Wylezich, C., Herlemann, D. P. \& Jürgens, K. Improved $18 \mathrm{~S}$ rDNA amplification protocol for assessing protist diversity in oxygendeficient marine systems. Aquatic Microbial Ecology 81, 83-94 (2018).

23. Van der Auwera, G., Chapelle, S. \& De Wächter, R. Structure of the large ribosomal subunit RNA of Phytophthora megasperma, and phylogeny of the oomycetes. FEBS letters 338, 133-136 (1994).

24. Moreira, D. et al. Global eukaryote phylogeny: combined small-and large-subunit ribosomal DNA trees support monophyly of Rhizaria, Retaria and Excavata. Molecular phylogenetics and evolution 44, 255-266 (2007).

25. Williams, A. G. Rumen holotrich ciliate protozoa. Microbiological reviews 50, 25-49 (1986).

26. Corliss, J. O. On the evolution and systematics of ciliated protozoa. Systematic Zoology 5, 68-91 (1956).

27. Taylor, M. Protozoal disease in cattle and sheep. In practice-london-british veterinary association 22, 604-626 (2000).

28. Branton, D. et al. In Nanoscience And Technology: A Collection of Reviews from Nature Journals 261-268 (World Scientific, 2010).

29. Buermans, H. \& Den Dunnen, J. Next generation sequencing technology: advances and applications. Biochimica et Biophysica Acta (BBA)-Molecular Basis of Disease 1842, 1932-1941 (2014).

30. Belda, E. et al. Preferential suppression of Anopheles gambiae host sequences allows detection of the mosquito eukaryotic microbiome. Scientific reports 7, 3241, https://doi.org/10.1038/s41598-017-03487-1 (2017).

31. Comeau, A. M., Douglas, G. M. \& Langille, M. G. Microbiome helper: a custom and streamlined workflow for microbiome research. mSystems 2, e00127-00116 (2017).

32. Vestheim, H., Deagle, B. E. \& Jarman, S. N. Application of blocking oligonucleotides to improve signal-to-noise ratio in a PCR. Methods in molecular biology (Clifton, N.J.) 687, 265-274, https://doi.org/10.1007/978-1-60761-944-4_19 (2011). 
33. Cannon, M. V. et al. A high-throughput sequencing assay to comprehensively detect and characterize unicellular eukaryotes and helminths from biological and environmental samples. Microbiome 6, 195, https://doi.org/10.1186/s40168-018-0581-6 (2018).

34. Afrin, T., Kounosu, A., Billah, M.-M., Murase, K. \& Kikuchi, T. Evaluation of magnetic cellulose bead-based DNA extraction from faecal materials for high-throughput bacterial community analyses. Applied Entomology and Zoology 53, 281-286 (2018).

35. Caporaso, J. G. et al. QIIME allows analysis of high-throughput community sequencing data. Nature methods 7, 335-336, https:// doi.org/10.1038/nmeth.f.303 (2010).

36. Quast, C. et al. The SILVA ribosomal RNA gene database project: improved data processing and web-based tools. Nucleic acids research 41, D590-596, https://doi.org/10.1093/nar/gks1219 (2013).

37. Oksanen, J. et al. Vegan: community ecology package. R package version 1.17-4, http://cran.r-project.org. Acesso em 23, 2010 (2010).

\section{Acknowledgements}

We thank Akina Hino, Tanzila Afrin, Ryusei Tanaka, Vicky Hunt, Mark Bligh, Mana Abe and Yasunobu Maeda for helpful comments and support and Aya Adachi for technical assistance.

\section{Author contributions}

H.M. and T.K. conceived and designed the study. A.K. and A.Y. performed experiments. A.K., K.M. and T.K. analysed data. K.A. and T.K. wrote the manuscript from input from K.M. All authors reviewed and approved the manuscript.

\section{Competing interests}

The authors declare no competing interests.

\section{Additional information}

Supplementary information is available for this paper at https://doi.org/10.1038/s41598-019-52422-z.

Correspondence and requests for materials should be addressed to T.K.

Reprints and permissions information is available at www.nature.com/reprints.

Publisher's note Springer Nature remains neutral with regard to jurisdictional claims in published maps and institutional affiliations.

(c) (i) Open Access This article is licensed under a Creative Commons Attribution 4.0 International License, which permits use, sharing, adaptation, distribution and reproduction in any medium or format, as long as you give appropriate credit to the original author(s) and the source, provide a link to the Creative Commons license, and indicate if changes were made. The images or other third party material in this article are included in the article's Creative Commons license, unless indicated otherwise in a credit line to the material. If material is not included in the article's Creative Commons license and your intended use is not permitted by statutory regulation or exceeds the permitted use, you will need to obtain permission directly from the copyright holder. To view a copy of this license, visit http://creativecommons.org/licenses/by/4.0/.

(C) The Author(s) 2019 